\title{
Previously differentiated medial vascular smooth muscle cells contribute to neointima formation following vascular injury
}

\author{
Vascular Cell 6:21 ｜ DOI: 10.1186/2045-824X-6-21 ～～C Li et al.; licensee Publiverse Online S.R.L. 2014 \\ Received: 11 Aug 2014 I Accepted: 10 Aug 2014 I Published: 1 Aug 2014 \\ Herring Brian Paul ${ }^{@}$, Hoggatt April M, Burlak Christopher, Offermanns Stefan \\ ${ }^{+}$Contributed equally ${ }^{\circledR}$ Corresponding author
}

\section{Abstract}

\section{Background}

The origins of neointimal smooth muscle cells that arise following vascular injury remains controversial. Studies have suggested that these cells may arise from previously differentiated medial vascular smooth muscle cells, resident stem cells or blood born progenitors. In the current study we examined the contribution of the previously differentiated vascular smooth muscle cells to the neointima that forms following carotid artery ligation.

\section{Methods}

We utilized transgenic mice harboring a cre recombinase-dependent reporter gene (mTmG). These mice express membrane targeted tandem dimer Tomato (mTomato) prior to cre-mediated excision and membrane targeted EGFP (mEGFP) following excision. The $\mathrm{mTmG}$ mice were crossed with transgenic mice expressing either smooth muscle myosin heavy chain ( Myh11) or smooth muscle $\alpha$-actin ( Acta2) driven tamoxifen regulated cre recombinase. Following treatment of adult mice with tamoxifen these mice express mEGFP exclusively in differentiated smooth muscle cells. Subsequently vascular injury was induced in the mice by carotid artery ligation and the contribution of mEGFP positive cells to the neointima determined.

\section{Results}

Analysis of the cellular composition of the neointima that forms following injury revealed that mEGFP positive cells derived from either Mhy 11 or Acta 2 tagged medial vascular smooth muscle cells contribute to the majority of neointima formation $(79 \pm 17 \%$ and $81 \pm 12 \%$, respectively).

\section{Conclusion}

These data demonstrate that the majority of the neointima that forms following carotid ligation is derived from previously differentiated medial vascular smooth muscle cells.

\section{Keywords}

Vascular smooth muscle - Neointima - Smooth muscle myosin - Smooth muscle $\alpha$-actin

\section{Background}

Vascular smooth muscle cells (VSMCs) are the major contractile components of the vascular system. They are critically important for regulating blood pressure and flow throughout the vascular system. Unlike skeletal and cardiac muscle cells, VSMCs are remarkably plastic and modulate their phenotype in response to extracellular cues during the development and progression of a variety of diseases including atherosclerosis, hypertension, stenosis following injury and restenosis following vascular interventions. Cardiovascular disease is the leading 
cause of death among the US population, yet despite intense research efforts a number of basic questions regarding the etiology of cardiovascular disease remain elusive. Classically these diseases are described as being associated with dedifferentiated VSMCs that have decreased expression of proteins required for the normal contractile function, increased expression of extracellular matrix proteins and increased cell proliferation [ 1]. These proliferating dedifferentiated VSMCs are a major component of neointimal lesions and atherosclerotic plaques. Neointimal VSMCs have been proposed to arise from several sources, including blood and bone marrow derived precursor cells, dedifferentiated medial VSMCs, resident progenitor cells and adventitial fibroblasts. However, recent definitive studies showed a relatively minor contribution of blood and bone marrow derived cells to the neointima or atherosclerotic plaque VSMC population [ 2-5]. The most widely accepted paradigm that neointimal VSMCs arise from the dedifferentiation and migration of medial VSMCs has been recently challenged [ 6]. This finding has stimulated much controversy in the field [ 7] and has prompted us to further investigate the origin of these cells. Using a genetic fate mapping approach with tamoxifen regulated smooth muscle-specific cre recombinase and a dual color cre-dependent reporter gene we unequivocally show that the neointimal SMCs that arise following carotid artery ligation are largely derived from the previously differentiated medial VSMCs.

\section{Methods}

\section{Transgenic mice and carotid ligation}

All animal procedures were performed using procedures approved by the Indiana University School of Medicine Institutional Animal Care and Use committee under protocol number 10310. Smooth muscle myosin heavy chain ( Myhl1 ) creER(T2) ${ }^{-/+}$mice [ 8] (on a C57BL6 background) and smooth muscle $\alpha$-actin ( Acta2 ) creER(T2) ${ }^{-/+}$mice [9] were crossed with $\mathrm{mTmG}$ reporter mice (Jackson strain: B6.129(Cg)-Gt(ROSA)26Sor ${ }^{\text {tm4(ACTB- }}$ tdTomato,-EGFP)Luo /J) (Figure 1). creER(T2) transgenes were used under agreement from the Institut de Génétique et de Biologie Moléculaire et Cellulaire (IGBMC). Double heterozygous transgenic mice were used for all experiments unless indicated otherwise. At 5-6 weeks of age mice were treated with Tamoxifen $(1 \mathrm{mg} / \mathrm{mouse}$ IP) or corn oil control, once a day for 5 days. 2 weeks following the last treatment the left carotid artery was ligated as described previously [ 10]. Prior to surgery mice were anesthetized with intraperitoneal injection of ketamine/xylazine $(0.088 \mathrm{mg} / \mathrm{gm}, 0.012 \mathrm{mg} / \mathrm{gm})$. Following surgery mice were given a single subcutaneous injection of carprofen analgesic $(0.5 \mathrm{mg})$. At various times following ligation mice were sacrificed under anesthesia (intraperitoneal injection of ketamine/xylazine $(0.088 \mathrm{mg} / \mathrm{gm}, 0.012 \mathrm{mg} / \mathrm{gm})$ and the injured and control contralateral carotid arteries were harvested and fixed in $4 \%$ paraformaldehyde for 2 hours on ice. Following fixation, vessels were washed in phosphate buffered saline for $3 \times 5$ minutes and then frozen in OCT tissue freezing media (Tissue-Tech) on a bed of dry ice/2-methylbutane. $8 \mu \mathrm{m}$ cryosections were cut and stored at $-80^{\circ}$.

Figure 1 


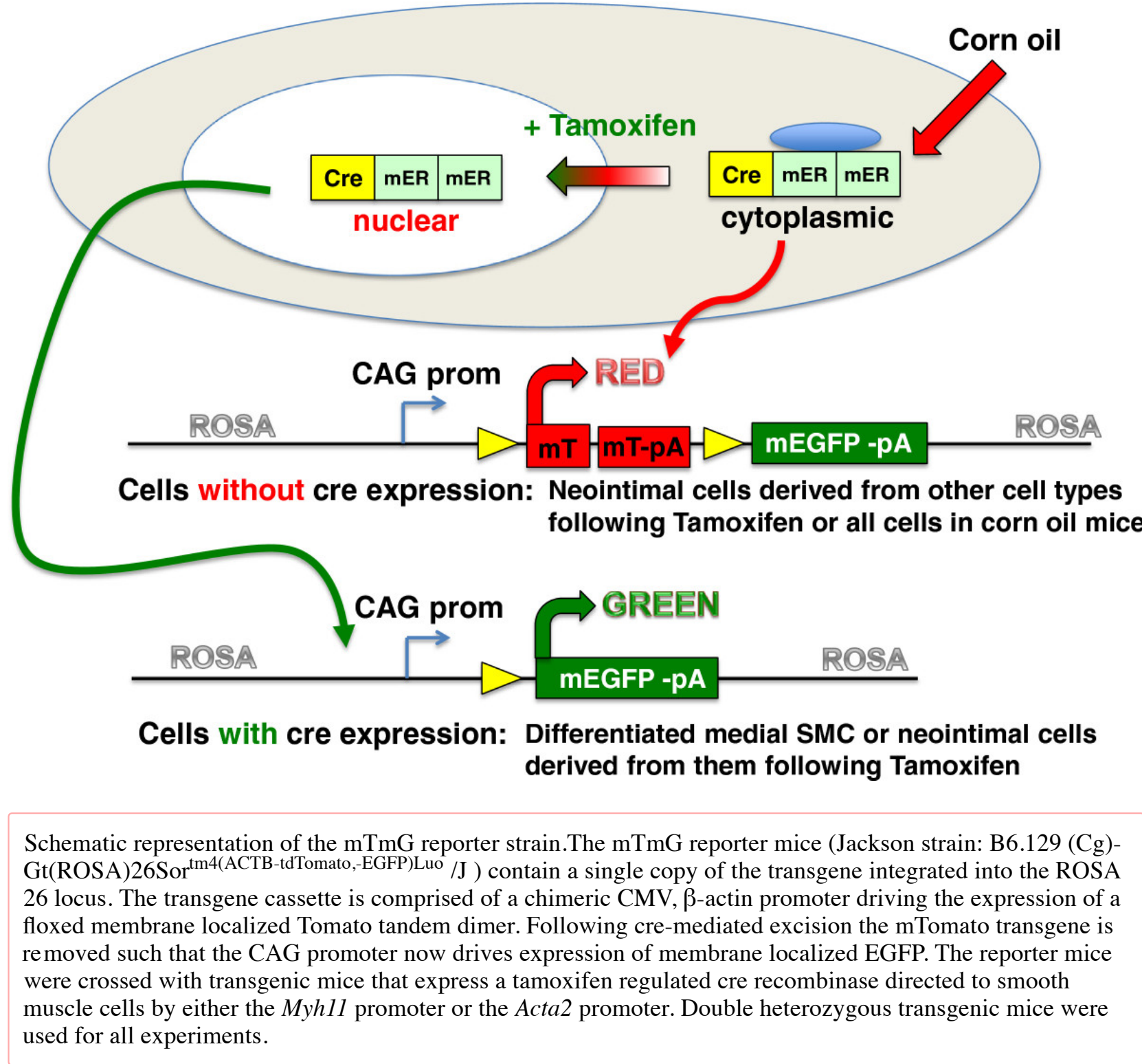

\section{Histological and immuno-staining}

OCT was removed from sections by 3, 5 minute washes in Tris buffered saline (TBS; $100 \mathrm{mM}$ Tris $\mathrm{pH} 7.6$, $150 \mathrm{mM} \mathrm{NaCl}$ ). Slides were mounted in Prolong Gold containing DAPI (Invitrogen) and visualized by confocal microscopy (Olympus Fluoview FV1000). For immuno-fluorescent staining cryosections were permeabilized in $0.2 \%$ triton in TBS for 5 minutes, washed in TBS for 5 minutes them blocked in 5\% goat serum diluted in TBS at room temperature for 1 hour. Blocked sections were incubated for $4-5$ hours at $37^{\circ}$ with primary antibodies to, the SM2 isoform of smooth muscle myosin heavy chain (1:500) [ 11], CD31 (1:100, clone 390, Affymetrix, eBioscience), CD68 (1:200, clone FA-11, AbD Serotec) diluted in 5\% goat serum/TBS. Some sections were incubated without primary antibody as a negative control. Following washing in TBS primary antibodies were detected by incubation with anti-rabbit or anti-rat Alexa Fluor 647 (1:10,000, Jackson ImmunoResearch). After washing slides were mounted in Prolong Gold containing DAPI (Invitrogen) and visualized by confocal microscopy.

\section{Quantitation of mEGFP and mTomato positive cells in lesions}

In order to count the number of mEGFP and mTomato positive cells within the neointima of each vessel, a Z-stack series of $1 \mu \mathrm{m}$ optical sections were obtained from each vessel. mEGFP and mTomato positive cells were counted in each of the optical sections through the entire Z-stack of images, each DAPI positive nuclei was scored as being associated with either an mEGFP or mTomato positive cell. For some sections it was possible to obtain 2 different fields of Z-stack images.

\section{Results}

Results of a recent study suggest that neointimal cells that form following vascular injury are derived from a stem cell population resident within the vascular wall rather than from previously differentiated VSMCs [ 6]. In that 
study fate mapping experiments were performed in which differentiated VSMCs were tagged using a credependent EGFP reporter strain crossed with transgenic mice expressing cre recombinase exclusively in smooth muscle cells (B6.Cg-Tg(Myh11-cre,-EGFP)2Mik/J) [ 12]. The conclusion that previously differentiated VSMCs do not contribute to neointima formation was thus, based largely on the inability to detect EGFP-positive cells in the neointima in these mice. As there are many factors that can contribute to a negative result in these experiments, including the reported silencing of the ROSA 26 promoter that was used to drive EGFP expression in neointimal cells [ 13], we reevaluated these findings using an alternative fate mapping strategy. We utilized a dual color reporter transgenic mouse line (mTmG: B6.129(Cg)-Gt(ROSA)26Sor ${ }^{\text {tm4(ACTB-tdTomato,-EGFP)Luo } / J) ~ i n ~}$ which all cells express a membrane localized mTomato tandem dimer in the absence of cre recombinase activity. Following cre-mediated excision of the mTomato cassette, cells express membrane localized EGFP [14] (Figure 1 ). In this reporter mouse line, both mTomato and mEGFP are driven by the same CMV/ $\beta$-actin (CAG) promoter that has been shown to be active in neointimal cells [ 13]. This dual color reporter strain obviates the need to interpret negative data, as all neointimal cells should be either red or green depending on whether they express mTomato of mEGFP, respectively. To specifically identify differentiated VSMCs we crossed the mTmG mice with mice expressing a tamoxifen regulated cre recombinase directed by the smooth muscle-specific Myhl1 [ 8] or Acta2 [ 9] promoters. The ability to temporally control cre recombinase activity also permitted us to distinguish previously differentiated VSMCs from newly differentiated VSMCs. Mice were treated with tamoxifen to activate the cre recombinase 2 weeks prior to vascular injury. As tamoxifen is rapidly metabolized in mice, at the time of surgical injury and subsequently, any cre recombinase expressed in VSMCs will be inactive and thus unable to switch cells from mTomato positive to mEGFP positive. Thus only VSMCs that were differentiated during the period of tamoxifen treatment, prior to injury will be mEGFP positive. As cre-mediated recombination results in a permanent heritable change in a cell's genome these cells and any of their progeny will remain mEGFP positive even if they dedifferentiate and loose expression of cre or Myhl1 or Acta2 .

In Myhll creER(T2) ${ }^{-/+} \mathrm{mTmG}^{-/+}$double transgenic mice [ 8] and Acta $2 \mathrm{creER}(\mathrm{T} 2)^{-/+} \mathrm{mTmG}^{-/+}$[ 9] double transgenic mice (Figure 1) treatment with tamoxifen results in cre mediated recombination and thus subsequent expression of mEGFP specifically in vascular and visceral smooth muscle cells (Figures 2 and 3 ). No mEGFP expression was observed in endothelial cells, adventitial cells, cardiac or skeletal muscle cells (Figures 2 and 3 ). 7 days following injury in $M y h 11 \mathrm{creER}(\mathrm{T} 2)^{-/+} \mathrm{mTmG}^{-/+}$double transgenic mice we observed a significant decrease in expression of the endogenous Myhl1 gene within the medial layer of carotid arteries as evidenced by decreased smooth muscle myosin isoform, SM2, immunostaining (Figure 4). This is consistent with the previously reported dedifferentiation of medial VSMCs that follows vascular injury [ 15]. Despite the observed decreased Myhll expression, the medial mEGFP expression was similar in control and injured vessels 7 days following injury, suggesting that the CAG promoter, which drives mEGFP expression, is not affected by injury (Figure 4). At this time point we did not observe any significant neointima formation in any of the three mice examined. In contrast, 14 days following ligation we observed small amounts of neointima formation in 3 out of 4 mice examined (Figure 5). In these mice, mEGFP positive cells can be readily seen within the neointima suggesting that these cells are derived from previously differentiated ( Myhl1 positive) medial VSMCs. Immunostaining with antibodies to CD31 demonstrate that there are also a number of CD31 positive, mTomato positive endothelial like cells within the neointima (Figure 6). We also observed some CD68 positive macrophage/monocytes in the neointima of some mice although these appeared less abundant than the CD31 positive cells (Figure 7 ). Figure 2

Tissue specificity of Myh11 cre ER(T2) activity.5-week old Myh11 creER(T2) ${ }^{-1+} \mathrm{mTmG}^{-/+}$mice were treated with tamoxifen ( $1 \mathrm{mg}, \mathrm{IP})$ once a day for 5 days. 6 weeks later tissues were harvested and analyzed by confocal microscopy as described in 'Methods'. A strong mEGFP signal can be seen only in smooth muscle cells of all the tissues examined including, bladder, colon, aorta, skeletal muscle and heart. No cre activity was detected in other cell types including skeletal muscle cells cardiac myocytes, endothelial cells or mucosal epithelial cells. SMsmooth muscle, LM-longitudinal muscle, CM-circular muscle, MP-myenteric plexus, MM-muscularis mucosa, EC-endothelial cells.

\section{Figure 3}

Tissue specificity of Acta2 cre ER(T2) activity.5-week old Acta 2 creER(T2) $)^{-/+} \mathrm{mTmG}^{-1+}$ mice were treated with tamoxifen ( $1 \mathrm{mg}, \mathrm{IP})$ once a day for 5 days. 2 weeks following the last tamoxifen injection the left carotid artery was ligated. 4 weeks later tissues were harvested and analyzed by confocal microscopy as described in 'Methods'. A strong mEGFP signal can be seen only in smooth muscle cells of all the tissues examined. No cre activity was detected in other cell types including skeletal muscle cells, cardiac myocytes, endothelial cells, mucosal epithelial cells and hepatocytes. A heterogeneous staining was observed in the uterus suggesting that cre was not active in all the uterine smooth muscle cells during the period in which the mice were treated with tamoxifen. The patchwork mEGFP expression observed in the esophagus reflects the mixed skeletal/smooth muscle lineage of cells within the wall of this portion of the esophagus. SM-smooth muscle, LM-longitudinal muscle, CM-circular muscle, MP-myenteric plexus, MM-muscularis mucosa. Scale bars represent $40 \mu \mathrm{m}$ in all panels.

Figure 4 
Myh11 expression is down-regulated 7 days following injury.5-week old male $M y h 11 \mathrm{creER}(\mathrm{T} 2)^{-/+} \mathrm{mTmG}^{-/+} \mathrm{mice}^{-}$ were treated with tamoxifen $(1 \mathrm{mg}, \mathrm{IP})$ once a day for 5 days. Two weeks following the last tamoxifen injection the left carotid artery was ligated and tissues were harvested 7 days later. $8 \mu \mathrm{m}$ cryosections obtained from injured and contralateral control arteries were analyzed for expression of myhl1 (using an anti-SM2 antibody) (red) and mEGFP (green) and mTomato (white). Control and injured sections are shown at identical exposures. Images shown are representative of those obtained from 3 different mice. In the lower panels the SM2 antibody was omitted and the samples otherwise processed identically to those shown in the upper panels. All images are shown at the same magnification with the scale bar shown in the bottom left panel representing $50 \mu \mathrm{m}$.

Figure 5

Previously differentiated VSMC contribute to early neointima formation.5-week old male Myh11 creER(T2) ${ }^{-/+} \mathrm{mTmG}^{-/+}$mice were treated with tamoxifen $(1 \mathrm{mg}, \mathrm{IP})$ once a day for 5 days. Two weeks following the last tamoxifen injection the left carotid artery was ligated and tissues were harvested 14 days later. Expression of mTomato (red) and mEGFP (green) were visualized in sections obtained from 4 different mice. Nuclei were visualized by staining with DAPI (blue). All images are shown at the same magnification with the scale bar shown in the top left panel representing $50 \mu \mathrm{m}$.

\section{Figure 6}

CD31 positive endothelial cells can be seen within early developing neointima.Sections from two of the mice shown in Figure 5 were stained with antibodies to CD31 (white). Left panels show mTomato (red)/mEGFP (green) co-stained sections and in the right panels are the same sections visualized for mEGFP (green) and CD31(white). Nuclei were visualized by staining with DAPI (blue). All images are shown at the same magnification with the scale bar shown in the bottom left panel representing $50 \mu \mathrm{m}$.

\section{Figure 7}

CD68 positive macrophages/monocytes cells can be seen within early developing neointima in some mice.Sections from the same two mice shown in Figure 6 were stained with antibodies to CD68 (white). Left panels show mTomato (red)/mEGFP (green) co-stained sections and in the right panels are the same sections visualized for mEGFP (green) and CD68(white). CD68-positive cells can be seen within the neointima of vessels from the mouse shown in the upper panels but not in the vessels of the mouse shown in the lower panels. Nuclei were visualized by staining with DAPI (blue). All images are shown at the same magnification with the scale bar shown in the bottom left panel representing $50 \mu \mathrm{m}$.

To better quantitate the contribution of mEGFP positive cells to neointima formation we examined more mature lesions that formed 28 days following ligation. In most $M y h 11 \mathrm{creER}(\mathrm{T} 2)^{-/+} \mathrm{mTmG}^{-/+}$double transgenic mice 28 days following carotid ligation the majority of neointimal cells also expressed mEGFP (Figure 8, Table 1). However, in one mouse the neointima was comprised of only $42 \%$ of mEGFP positive cells (Mouse\#6 in Table 1, Figure 9B, C). In this mouse the lesion was very unusual in that it extended out in a bulb like structure from one side of the vessel wall (Figure 9B). Many of the mTomato positive cells in this lesion also stained positive for either CD31 or CD68 suggesting a large number of endothelial cells and macrophages or monocytes in the lesion (Figure 9B, C). In contrast, no CD68 positive cells were detected in most mature lesions and only a few scattered endothelial cells were seen within these lesions, although they could be readily seen lining the residual vessel lumens (Figure 9A). In another mouse, not shown in Table 1, we did not observe any concentric neointima formation 28 days following injury. In contrast, in this mouse there was an mEGFP negative cluster of CD31 positive cells that covered approximately $50 \%$ of the vessel lumen. As this structure occurred close to the point of ligation ( $<100 \mu \mathrm{m}$ away), was very focal only extending for about $300 \mu \mathrm{m}$, was atypical in shape and comprised of CD31 positive cells we speculate that it was an organized thrombus rather than a true neointimal lesion. This mouse was thus excluded from our analyses. In control mice, given corn oil instead of tamoxifen, almost all cells remained mTomato positive and mEGFP negative (Figure 8 , lower right panel). One mEGFP positive cell can be seen in the media of the corn oil treated vessel (green/yellow cell indicated by arrow head). This cell likely represents one in which the creER(T2) protein has moved to the nucleus in the absence of the tamoxifen ligand. As this cell also seems to express some mTomato (accounting for its yellow color) it is likely that the recombination event occurred within a few days of tissue harvesting such that the residual mTomato protein has not had time to completely turn over and disappear from the cell. Figure 8

Myhl1 -tagged medial SMCs give rise to the mature neointima following carotid ligation.Tissues were harvested 28 days following carotid ligation of 5-week old male Myhll creER(T2) $)^{-1+} \mathrm{mTmG}^{-1+}$ mice that were previously Treated with Tamoxifen (TAM) or corn oil(CO) as indicated. In the upper two panels the control and injured carotid arteries from the same mouse are shown. The neointima, media and adventitia (adv) are indicated. Images are representative of those obtained from 7 tamoxifen treated mice (Mice numbers 1,7,2,3 in Table 1, as indicated). Arrows point to examples of endothelial cell nuclei. The image in the lower right hand panel is of an injured vessel obtained from a corn oil control treated mouse. The arrow-head in this image points to an mEGFP positive cell within the medial layer. All images are shown at the same magnification with the scale bars representing $50 \mu \mathrm{m}$. 
Consistent with data obtained from the Myhl1 cre mice, when previously differentiated VSMCs were tagged with the use of Acta2 cre the majority of the neointimal cells were mEGFP positive (range 63-94\%; mean of $81 \pm 12 \%$; Figure 10, Table 1). Although the data presented suggest that many neointimal cells are derived from previously differentiated ( Myhll or Acta2 positive) medial VSMCs it is possible that these cells may be mobilized from distant sites rather than being derived from locally resident medial VSMCs. To evaluate the existence of a population of mobile mEGFP positive cells we performed a fluorescence activated cell sorting (FACS) analysis of blood obtained from tamoxifen treated Myh11 creER(T2) ${ }^{-/+} \mathrm{mTmG}^{-/+}$double transgenic mice, 7,14 and 28 days following injury (Figure 11). This analysis failed to detect the presence of any significant numbers of mEGFP positive circulating cells suggesting that the mEGFP positive neointimal cells are most likely locally derived. Figure 10

Acta2 -tagged medial SMCs give rise to the neointima following carotid ligation.Control and injured carotid arteries from Tamoxifen (TAM) or corn oil (CO) treated Acta 2 creER(T2) $)^{-1+} \mathrm{mTmG}^{-/+}$transgenic mice harvested 28 days post ligation (Mice numbers 11,12 and 13, respectively; Table 1). Images from tamoxifen treated injured vessels are representative of those obtained from 7 mice.

Figure 11

No mEGFP positive circulating cells can be detected following carotid ligation.Blood was harvested from Myhl1 creER $(\mathrm{T} 2)^{-/+} \mathrm{mTmG}^{-/+}$mice 7,14 and 28 days following carotid ligation and subjected to fluorescence activated cell sorting. Cells harvested from a CAG-EGFP mouse were used as positive controls for EGFP expressing cells, those harvested from $\mathrm{mTmG}$ reporter mice with no cre transgene as positive controls for mTomato positive cells and those harvested from nontransgenic mice as negative controls. From each mouse a minimum of 10,000 cells were sorted. Upper panels show the distribution of mTomato (red) and mEGFP (green) positive as well as negative (black) cells. Numbers on each graph show the percentage of negative cells in each quadrant. Lower panels show the distribution of cells obtained from duplicate $M y h 11 \mathrm{creER}(\mathrm{T} 2)^{-/+} \mathrm{mTmG}^{-/+}$mice. In each case the percentage of EGFP-positive cells are less than background negative control levels.

\section{Discussion}

Results from the current studies support the widely accepted paradigm that following vascular injury medial VSMCs dedifferentiate and migrate into the lumen of vessels forming a neointima. As we utilized a tamoxifen regulated cre recombinase and waited 2 weeks after tamoxifen treatment before performing carotid ligation, given the 12 hour half life of tamoxifen in serum, it is highly unlikely that mEGFP positive cells seen following injury are derived from newly differentiated cells. Moreover, the use of a dual color reporter system avoided any artifacts that may arise due to promoter or reporter silencing as all cells should be either mTomato or mEGFP positive thus no conclusions need to be drawn that are based on negative staining data. Our data also suggest that unlike the ROSA-LACz reporter gene [ 13] the CMV enhancer/chicken beta-actin core promoter (CAG) driven mTmG reporter gene is not down-regulated in neointimal SMCs (compare the mEGFP intensity of control and injured vessels in Figures 3, 8 and 10). The use of the $\mathrm{mTmG}$ reporter strain has the additional advantage that the reporter proteins are membrane localized and thus better retained during sample processing. Moreover, this is a single copy, targeted transgene, hence, it is also not subject to complications that may arise from partial recombination of multicopy transgenes, such that in each cell's nucleus either the mTomato gene is present or it is excised. Cells will thus express either mTomato or mEGFP. Anecdotally we have noted, that the mTomato protein is relatively stable such that after tamoxifen treatment smooth muscle cells can have detectable expression of both mTomato and mEGFP for 3-4 days before the mTomato protein is turned over and degraded. We speculate that one or more of these advantages of the $\mathrm{mTmG}$ reporter system and tamoxifen regulated cre transgenes used in our study may explain why we were able to detect mEGFP positive neointimal cells whereas they were not detected in a previous study [6].

Our data are consistent with and extend previous fate mapping studies using a cre-dependent LacZ reporter [ 5]. In this study a cre-dependent ROSA-LacZ reporter was used together with Myhl1-creER(T2) transgenic mice to show that following femoral artery wire injury the neointima that formed contained LacZ positive cells [ 5]. Together these studies suggest that in both the small concentric lesions that form following femoral wire injury and the more complex large lesions that form following carotid ligation, neointimal cells arise from differentiated medial VSMCs. Our rigorous quantitative analysis using $1 \mu \mathrm{m}$ optical sections to obtain Z-stack series through the entire thickness of each $8 \mu \mathrm{m}$ section, revealed some heterogeneity between mice, with mEGFP positive cells comprising $42-94 \%$ of total neointimal cells (Table 1). Although most mice have between 70-90\% mEGFP positive neointimal cells, this may perhaps account for some of confusion in the literature related to the contribution of different cell types to the neointima. Despite this variability, the data indicate that, on average, the majority $(\sim 80 \%)$ of neointimal cells arise from the previously differentiated medial SMCs. This number would be consistent with previous studies that showed that about $20 \%$ of neointimal cells are derived from blood borne cells [ 4]. In further support of the contribution of blood or endothelial derived cells to the neointima some of the mTomato positive, mEGFP negative neointimal cells observed in our study, stained positive for endothelial and monocyte markers (CD31 and CD68, respectively; Figures 6,7 and 9). It is perhaps a little surprising that in 
some lesions there were more CD31 positive endothelial cells within the lesions than CD68 positive monocytes/macrophages. Some of these CD31 positive endothelial cells may be present in new vessels that are growing within the neointima (e.g. Figure 6 , lower panels) and some may be miscounted lumen endothelial cells in which the plane of the section has obscured a luminal invagination. We also speculate that the proliferating neointimal smooth muscle cells may trap endothelial cells within the lesion as they extend out into the vessel lumen.

\section{Conclusions}

Although our studies do not rule out the possibility that under appropriate, in vitro, culture conditions the expansion of a progenitor cell population may be favored, the current studies provide compelling evidence that, in vivo, the majority of neointimal cells that arise following carotid ligation are derived from differentiated medial VSMCs. The lack of detectable mEGFP positive cells circulating in the blood, further suggests that the neointimal cells likely arise from the dedifferentiation and migration of locally derived medial VSMCs.

\section{Declarations}

\section{Acknowledgements}

This research was supported by research support funds from IUPUI.

\section{Authors' original submitted files for images}

Below are the links to the authors' original submitted files for images.

Authors' original file for figure 1

Authors' original file for figure 2

Authors' original file for figure 3

Authors' original file for figure 4

Authors' original file for figure 5

Authors' original file for figure 6

Authors' original file for figure 7

Authors' original file for figure 8

Authors' original file for figure 9

Authors' original file for figure 10

Authors' original file for figure 11

\section{Competing interests}

The authors declare that they have no competing interests.

\section{Authors' contributions}

BPH prepared the manuscript and figures, harvested tissues, obtained cryosections, performed all confocal microscopy, immunohistology and quantitative analysis. AMH maintained mouse lines, performed carotid ligations and harvested tissues. CB provided access and training in the use of the confocal microscope and performed FACS studies. SO generated the myhl1 creER(T2) transgenic mice, aided in data interpretation and reviewed and edited the final manuscript. All authors reviewed and approved the final manuscript.

\section{References}

1. Dandre F, Owens GK. Platelet-derived growth factor-BB and Ets-1 transcription factor negatively regulate transcription of multiple smooth muscle cell differentiation marker genes. Am J Physiol Heart Circ Physiol. 2004;286:H2042-H2051.

View Article Google Scholar 
2. Hu Y, Davison F, Ludewig B, Erdel M, Mayr M, Url M, Dietrich H, Xu Q. Smooth muscle cells in transplant atherosclerotic lesions are originated from recipients, but not bone marrow progenitor cells. Circulation. 2002;106:1834-1839.

View Article Google Scholar

3. Bentzon JF, Sondergaard CS, Kassem M, Falk E. Smooth muscle cells healing atherosclerotic plaque disruptions are of local, not blood, origin in apolipoprotein E knockout mice. Circulation. 2007;116:2053-2061.

View Article Google Scholar

4. Iwata H, Manabe I, Fujiu K, Yamamoto T, Takeda N, Eguchi K, Furuya A, Kuro-o M, Sata M, Nagai R. Bone marrow-derived cells contribute to vascular inflammation but do not differentiate into smooth muscle cell lineages. Circulation. 2010;122:2048-2057.

View Article Google Scholar

5. Nemenoff RA Horita H Ostriker AC Furgeson SB Simpson PA VanPutten V Crossno J Offermanns S Weiser-Evans MC SDF-1alpha induction in mature smooth muscle cells by inactivation of PTEN is a critical mediator of exacerbated injury-induced neointima formation Arterioscler Thromb Vasc Biol 201131130013083111081 10.1161/ATVBAHA.111.223701

6. Tang Z Wang A Yuan F Yan Z Liu B Chu JS Helms JA Li S Differentiation of multipotent vascular stem cells contributes to vascular diseases Nat Commun 201238753538044 $10.1038 /$ ncomms 1867

7. Nguyen AT Gomez D Bell RD Campbell JH Clowes AW Gabbiani G Giachelli CM Parmacek MS Raines EW Rusch NJ Speer MY Sturek M Thyberg J Towler DA Weiser-Evans MC Yan C Miano JM Owens GK Smooth muscle cell plasticity: fact or fiction? Circ Res 201311217224135725 10.1161/CIRCRESAHA.112.281048

8. Wirth A, Benyo Z, Lukasova M, Leutgeb B, Wettschureck N, Gorbey S, Orsy P, Horvath B, Maser-Gluth C, Greiner E, Lemmer B, Schutz G, Gutkind JS, Offermanns S. G12-G13-LARG-mediated signaling in vascular smooth muscle is required for salt-induced hypertension. Nat Med. 2008;14:64-68.

View Article Google Scholar

9. Wendling O, Bornert JM, Chambon P, Metzger D. Efficient temporally-controlled targeted mutagenesis in smooth muscle cells of the adult mouse. Genesis. 2009;47:14-18.

View Article Google Scholar

10. Kumar A, Lindner V. Remodeling with neointima formation in the mouse carotid artery after cessation of blood flow. Arterioscler Thromb Vasc Biol. 1997;17:2238-2244.

View Article Google Scholar

11. Gallagher PJ Jin Y Killough G Blue EK Lindner V Alterations in expression of myosin and myosin light chain kinases in response to vascular injury Am J Physiol Cell Physiol 2000279 C1078 C10872824508

12. Xin HB, Deng KY, Rishniw M, Ji G, Kotlikoff MI. Smooth muscle expression of Cre recombinase and eGFP in transgenic mice. Physiol Genomics. 2002;10:211-215.

View Article Google Scholar

13. Cuttler AS LeClair RJ Stohn JP Wang Q Sorenson CM Liaw L Lindner V Characterization of Pdgfrb-Cre transgenic mice reveals reduction of ROSA26 reporter activity in remodeling arteries Genesis 2011496736803244048 10.1002/dvg.20769

14. Muzumdar MD, Tasic B, Miyamichi K, Li L, Luo L. A global double-fluorescent Cre reporter mouse. Genesis. 2007;45:593-605.

View Article Google Scholar

15. Owens GK, Kumar MS, Wamhoff BR. Molecular regulation of vascular smooth muscle cell differentiation in development and disease. Physiol Rev. 2004;84:767-801. 
View Article Google Scholar 